\title{
NONLINEAR ADAPTIVE FILTERING FOR ECHO CANCELLATION
}

\author{
John F. Michaelides ${ }^{1,3}$ and P. Kabal ${ }^{1,2}$ \\ ${ }^{1}$ Electrical Engineering \\ McGill University \\ Montreal, Quebec H3A 2A7 \\ ${ }^{3}$ Bell-Northern Research
Verdun, Quebec H3E $1 \mathrm{H} 6$ \\ INRS-Télécommunications \\ Université du Québec \\ Verdun, Quebec H3E 1 H6
}

\begin{abstract}
This paper examines the problem of nonlinear adaptive filtering for echo cancellation. The high speed requirements in digital subscriber loops and voiceband data modems place constraints on the design of adaptive echo cancellers due to the presence of nonlinearities This paper considers table look-up structures and nonlinear filters based on the Volterra series for general nonlinearities, and nonlinear compensators for specific practical configurations. For the first category, means to speed up the initial convergence of an adaptive table look-up structure are suggested. The new configurations invoive two table driven structures, one for cancellation and one to form the reference signal. It is shown how the second structure can also be used for decision feedback equalization. A combined linea and nonlinear structure with improved convergence behaviour is also proposed. New theoretical convergence rate results are presented for such structures. In the second category, nonlinear compensators are cascaded with linear filters to combat nonlinearities for specific channel models.
\end{abstract}

\section{Introduction}

Echo cancellation is a method of realizing full-duplex digital transmission over a common medium. It finds application in both digital subscriber loops and in voiceband data modems. In fullduplex transmission, echoes from the data signal transmitted in one direction can interfere with the data signal flowing in the opposite direction unless these two data signals are in non-overlapping frequency bands.

Full-duplex transmission over two wire lines (without frequency separation) can be achieved by using a hybrid $(H)$ which functions as a four-wire to two-wire interface. A typical configuration of a full-duplex transmission system using echo cancellation is shown in Fig. 1 (the two-wire line may include a four-wire trunk facility in the middle of the connection in the case of voiceband transmission). When viewed from the "near" end, the imperfect isolation of the local hybrid gives rise to near-end interference, while the hybrid at the other end of the connection gives rise to far-end echo. The nearend echo $e_{k}$ is the interference due to the transmitted signal which appears at the input to the receiver as a result of the imbalance of the local hybrid circuit. The echo signal $e_{k}$ appears along with the far-end received signal $u_{k}$ and the additive noise $n_{k}$.

An echo cancelier is an adaptive filter that synthesizes an estimate of the echo by adapting its impulse response to the impulse response of the echo channel. The estimated echo $\hat{e}_{k}$ is subtracted from the received signal $s_{k}$. For the maximum far-end signal attenuation encountered in typical common carrier applications, the near-end echo must be attenuated at least $50-60 \mathrm{~dB}$ in order to result in an acceptable signal-to-echo interference ratio at the receiver input. Because of the large degree of cancellation required for data transmission the compensation of nonlinearities becomes an important consideration. The primary sources of nonlinearity are the data converters, the transmitted pulse asymmetry and the saturation of transformers. Several methods have been proposed for nonlinear echo cancellation. Two considered here, which can be used to compensate for general nonlinearities, are the "table look-up" method [1] and one based on a Volterra series expansion [2].

When the table look-up structure is compared with a linear transversal filter it is found that it converges at a much slower rate This is one of the major limitations of the table look-up structure. A multi-memory structure was introduced in [3] that offers faster convergence times. However cancellation of nonlinearities is limited in time span and a residual nonlinearity may remain. One of the aims of this paper is to introduce structures that offer faster convergence times than the table look-up structure as well as retaining the ability to cancel the full time span of the nonlinear effects. Also, recognizing the fact that in practice nonlinear channels may arise from specific causes, nonlinear compensators for specific practical configurations will be considered

\section{The Two-Memory Structure}

The presence of the far-end signal in the control signal that is used for echo canceller adaptation is a limiting factor in the convergence rate. Because of the disturbing effect of the far-end signal on the adaptation algorithm, conventional echo cancellers (using linear FIR filters) use a very small stepsize to average out the effect of interference during full-duplex transmission. This has the side effect of causing the canceller to converge relatively slowly To overcome this effect, adaptive reference echo cancellation is used [4].

With adaptive reference echo cancellation, a reference signal which is an estimate of the far-end signal is formed by a referenceformer, and is subtracted from the control signal of the echo canceller adaptation algorithm. The reference-former used in [4] was a linear FIR filter. In this paper the reference-former uses a table look-up procedure (see Fig. 2)

2.1 Echo Canceller Configuration

The purpose of the second memory is to adaptively form a reference signal $\hat{u}_{k}$ which is an estimate of $u_{k}$. The reference signal $\hat{u}_{k}$ is then subtracted from the signal $r_{k}$. The resulting signal $r_{k}^{\prime}$ is then used for the adaptation of the echo canceller (RAM1) and of the reference-former (RAM2). Thus the table look-up structure for the reference-former can also compensate for any nonlinearities present in the communication channel which distort the received signal, and limit the degree of cancellation that can be reached if a linear filter is used.

The number of digits used to address the echo canceller is chosen so as to span the longest expected echo impulse response.

30.3.1. 
Similarly, the number of digits used to address the reference-former is chosen to span the longest expected communication channe impulse response. A delay may be included before subtraction of the reference-former signal $\hat{u}_{k}$ from the signal $r_{k}$ to account for delayed decisions due to the receiver decoder

\subsection{Adaptation Algorithms}

Two algorithms that could be used to adapt the two memories are the stochastic iteration algorithm (SIA), and the sign algorithm (SA). The adaptation equations are given by

$$
\begin{array}{lll}
\text { RAM1 : } & \hat{e}_{k+1}=\hat{e}_{k}+\alpha_{c} r_{k}^{\prime} & \text { SIA } \\
& \hat{e}_{k+1}=\hat{e}_{k}+\alpha_{C} \operatorname{sgn}\left(r_{k}^{\prime}\right) & \text { SA } \\
\text { RAM2 : } & \hat{u}_{k+1}=\hat{u}_{k}+\alpha_{n} r_{k}^{\prime} & \text { SIA } \\
& \hat{u}_{k+1}=\hat{u}_{k}+\alpha_{n} \operatorname{sgn}\left(r_{k}^{\prime}\right) & \text { SA }
\end{array}
$$

where $\alpha_{C}$ and $\alpha_{n}$ are the stepsizes used for the echo canceller and the reference-former respectively. In this paper we deal only with the SIA.

2.3 Convergence using the Stochastic Iteration Algorithm

Define the mean-square errors as,

$$
\varepsilon_{c, k}^{2} \triangleq E\left[\left(e_{k}-\hat{\epsilon}_{k}\right)^{2}\right], \quad \varepsilon_{n, k}^{2} \triangleq E\left[\left(u_{k}-\hat{u}_{k}\right)^{2}\right] .
$$

It can be shown [5] that the relation governing the convergence of the echo canceller is given by

$$
\varepsilon_{c, k+1}^{2}=\varepsilon_{c, k}^{2} \rho_{C}+\frac{\alpha_{C}^{2}}{2^{N_{C}}} \varepsilon_{n, k}^{2}+\frac{\alpha_{C}^{2}}{2^{N_{C}}} \sigma_{n}^{2},
$$

and for the reference-former is given by

$$
\varepsilon_{n, k+1}^{2}=\varepsilon_{n, k}^{2} \rho_{n}+\frac{\alpha_{n}^{2}}{2^{N_{n}}} \varepsilon_{c, k}^{2}+\frac{\alpha_{n}^{2}}{2^{N_{n}}} \sigma_{n}^{2},
$$

where

$$
\rho_{C}=1-\frac{\alpha_{c}\left(2-\alpha_{C}\right)}{2^{N_{C}}}, \quad \rho_{n}=1-\frac{\alpha_{n}\left(2-\alpha_{n}\right)}{2^{N_{n}}} .
$$

Notice that the convergence of the echo canceller is dependent on the error signal of the reference-former. The steady state value for the adaptive reference cancellation scheme is

$$
\varepsilon_{c, \infty}^{2}=\frac{\alpha_{c} \sigma_{n}^{2}}{2-\alpha_{c}-\alpha_{n}} .
$$

There is little penalty in making $\alpha_{n}=\alpha_{c}$ since small changes of $\alpha_{n}$ do not affect the value of $\varepsilon_{c, \infty}^{2}$ very much (as long as $\alpha_{n}$ is greater than zero, the steady state value reached with two memories will be lower than with one memory)

\section{Combined Echo Cancellation and Decision Feedback Equalization}

In Section 2, a second memory was added to the echo canceller configuration so that it could cancel the far-end data signal from the control signal used in the adaptation of the echo canceller. A third memory can be used as a DFE (decision feedback equalizer) but this would increase complexity and cost. Thus, in this section the reference-former (RAM2) serves a second role as part of a DFE (with the addition of some simple arithmetic operations).

3.1 Relation between the memory contents of the RAM

and the Volterra coefficients

In the previous section the adaptive reference-former is used as a means of identifying the communication channel. If the communication channel is modelled as an FIR filter then this results in the identification of the tap coefficients which are the impulse response coefficients of the channel. A subset of the impulse response coefficients cause intersymbol interference (ISI) from previously detected far-end symbols. When a table look-up structure is used the impulse response coefficients are not directly available. The goal here is to relate these coefficients to the memory contents of the RAM and use them to cancel intersymbol interference.

Starting from the Volterra series expansion, a new representation of the input 'output relationship of a nonlinear channel can be found in the case of binary signals $( \pm 1)[2]$. This representation has a finite number of terms and is given by

$$
\begin{aligned}
& f\left(b_{k}, \ldots, b_{k-N+1}\right)=g^{(0)}+\sum_{i=0}^{N-1} g_{i}^{(1)} b_{k-2}+\sum_{2_{1} \neq i_{2}}^{N-1} g_{i_{3}, i_{2}}^{(2)} b_{k-2_{1}} b_{k-i_{2}} \\
& +\cdots+\sum_{i_{1} \neq i_{2} \cdots \neq i_{N-1}}^{N-1} g_{2_{1}, i_{2}, \ldots, i_{N-1}}^{(N-1)} b_{k-1, b_{k-i_{2}} \ldots b_{k-i_{N-1}}} \\
& +g^{(N)} b_{k} b_{k-1} \ldots b_{k-N+1}
\end{aligned}
$$

where $f\left(b_{k}, \ldots, b_{k-N+1}\right)$ represents the contents of one of the $2^{N}$ memory locations of the RAM and the $g$ 's represent the Volterra coefficients in the new representation. The address of the RAM is specified by $b_{k}, b_{k-1}, \ldots, b_{k-N+1}$. The relationship between the contents of the memory of the RAM and the Volterra coefficients is found by assigning all the combinations of $b_{k}, b_{k-1}, \cdots, b_{k-N+1}$ in Eq. (7). The matrix resulting from this relation is orthogonal and its inverse is its transpose under an appropriate normalization. Thus the memory contents of the RAM can be found from the Volterra coefficients and vice-versa. If the communication channel is linear and modelled as an FIR filter, the Volterra coefficients $g_{0}^{(1)}, \ldots, g_{N-1}^{(1)}$ are the same as the tap coefficients of the filter and the remaining Volterra coefficients $g^{(0)}, g_{0,1}^{(2)}, \ldots, g^{(N)}$ are zero

It should be noted that $\mathrm{Eq}$. (7) represents the most general case and that it depends only on $N$ (the memory length) and not on $n$ (the order of nonlinearity). In practical situations some terms in $\mathrm{Eq}$. (7) may be essentially zero. If the order of the nonlinearity is smaller than $N$, the number of terms in the representation is

$$
N_{\text {terms }}=\sum_{M=0}^{n}\left(\begin{array}{l}
N \\
M
\end{array}\right), \quad n<N .
$$

\subsection{DFE Configuration}

In this section the relationship between the memory contents of the RAM and the Volterra coefficients is used to determine the DFE output values. From Eq. (7) we can see that the reference-former is trying to identify the Volterra coefficients $g^{(0)}, g_{0}^{(1)}, \cdots, g^{\left(N_{n}\right)}$ where $N_{R}$ is the memory length of the communication channel. The DFE tries to cancel all the terms of Eq. (7) except $g_{0}^{(1)} b_{k}$ and the cross product terms that require knowledge of the current value of $b_{k}$. Given the contents of RAM2, Eq. (7) can be used to determine the coefficients $g^{(0)}, g_{0}^{(1)}, \cdots, g^{\left(N_{R}\right)}$. Then using previously detected symbols the ISI component can be formed and subtracted from the current received signal before detection.

The configuration of the DFE is shown in Fig. 3. The figure only shows the DFE part. The rest of the transmission system is the same as in Fig. 2. The additional component is a processor that computes a subset of the Volterra coefficients. The processor does not need to perform any multiplications. Only additions and subtractions are required due to the simple relation that exists between the Volterra coefficients and the memory contents of the RAM. In general, the number of Volterra coefficients required is the number of terms that do not contain $b_{k}$. This is equal to

$$
\sum_{M=1}^{N_{n}}\left(\begin{array}{c}
N_{R}-1 \\
M-1
\end{array}\right)=2^{N_{\Pi}-1} .
$$

This number may be reduced by keeping only those terms that are significant. The equation that corresponds to $\mathrm{Eq}$. (8) for the 
DFE is

$$
N_{\text {terms }}=\sum_{M=0}^{n}\left(\begin{array}{c}
N_{R}-1 \\
M
\end{array}\right), \quad n<N_{R}
$$

If only second and third order nonlinearities are important and $N_{R}>3$ then from Eq. (10) the number of terms required to form $\tilde{u}_{k}$ is smaller than $2^{N_{n}-1}$. For example with $N_{R} \div 8$ only 64 terms are required to be computed by the processor whereas $2^{N_{n}-1}=128$. For non-time varying channels, the computation of the Volterra coefficients can be performed once, after the referenceformer has converged. For time varying channels, the $g$ 's can be continuously updated.

\section{The Combined Structure}

In this section a canceller structure is presented in which a linear FIR filter is added in parallel to the table look-up structure. The motivation for the addition of the FIR filter comes from convergence time considerations. The FIR filter can compensate for the linear part of the echo much faster than the table look-up structure. The table look-up structure is relegated to compensate for the nonlinear part. If the truncated echo impulse response is $N$ bits long then the FIR filter should have $N$ coefficients and the RAM should be addressed by $N$ bits. In fact, in the case of mild nonlinearities the RAM could be addressed by less than $N$ bits. This is justified from the fact that the bits transmitted last usually correspond to the largest nonlinear terms.

\subsection{Echo Canceller Configuration}

The new structure for the echo canceller is shown in Fig. 4. The total echo estimate is the sum of the echo estimates given by the FIR filter and the RAM as given below

$$
\begin{aligned}
\hat{e}_{k} & =\hat{e}_{1 k}+\hat{e}_{2 k} \\
& =\sum_{i=0}^{N-1} \hat{h}_{i k} a_{k-i}+f\left(a_{k}, a_{k-1}, a_{k-2}, \ldots, a_{k-N+1}\right) .
\end{aligned}
$$

where the $\left\{\hat{h}_{i k}\right\}$ represent the filter coefficients at time $k$. The nonlinear function $f(\cdot)$ can be represented by the Volterra series expansion.

The control signal used for the adaptation of both the FIR filter and the RAM is $r_{k}$. Consider a two-stage start-up method. In the first stage, the RAM is not activated. Thus during this time, the echo estimate is given by the first term $\hat{e}_{1 k}$ in Eq. 11. The FIR filter coefficients are updated using the stochastic iteration algorithm. When the FIR filter approaches its steady state, the second stage commences. The FIR coefficients are frozen, and the adaptation of the RAM coefficients begins (Alternately for time-varying channels, the FIR coefficients can continue to be adapted.) The steady state value reached will be that governed by the convergence equation for the table look-up structure [6] and consequently by the stepsize parameter used for the adaptation of the RAM coefficients.

\subsection{Convergence of the FIR filter}

It can be shown [5] that the convergence equation for a linear FIR filter for a nonlinear echo channel is given by

$$
R_{k}^{2}=\rho_{F}^{k}\left[R_{0}^{2}-\frac{\alpha_{F} N+2 G / \sigma_{u}^{2}}{2-\alpha_{F} N}\right]+\frac{\alpha_{F} N+2 G / \sigma_{u}^{2}}{2-\alpha_{F} N}
$$

where

$$
\rho_{F} \triangleq\left(1-2 \alpha_{F}+\alpha_{F}^{2} N\right),
$$

$R_{k}^{2}$ is the ratio of residual echo power to far-end data signal power and $\alpha_{F}$ is the stepsize parameter used for the adaptation of the filter coefficients. $G$ is a nonlinear factor representing the sum of the squares of the nonlinear terms of the Volterra coefficient vector. The nonlinear terms represent that part of the Volterra coefficient vector which the linear FIR filter cannot identify. The steady state value of $R_{k}^{2}$ is given by

$$
R_{\infty}^{2}=\frac{\alpha_{F} N+2 G / \sigma_{u}^{2}}{2} \text { for } \alpha_{k} \ll \frac{1}{N} .
$$

If we compare Eq. (13) with the case of a linear echo channel we find that there is a difference of $G / \sigma_{u}^{2}$ in the steady state value reached. For nonlinear channels, the nonlinear factor $G$ dominates the expression for the steady state value of the mean-square error and small values of the stepsize $\alpha_{F}$ do not bring much improvement. For a purely linear channel, $G$ becomes zero, and a small stepsize is useful in bringing down the mean-square error.

\section{A Nonlinear Compensator}

Both the table look-up structure and the Volterra series nonlinear filter can compensate for general nonlinearities. In practical situations however, nonlinear channels may take on a special form. Thus, specific nonlinear filters or compensators could be used instead of the general ones. In this section one such nonlinear compensator is examined, that is modelled in the same way as the channel that is trying to compensate for. The model consists of the cascade of a nonlinear part, a linear part and a second nonlinear part (Fig. 5).

The input/output relations at the different stages are

$$
\begin{aligned}
f_{1 k} & =\sum_{i=0}^{M-1} \alpha_{1 i} a_{k}^{i} \\
f_{2 k} & =\sum_{j=0}^{N-1} h_{j} f_{1 k-j}=\sum_{j=0}^{N-1} \sum_{i=0}^{M-1} h_{j} \alpha_{1 i} a_{k-j}^{i} \\
e_{k} & =\sum_{l=0}^{L-1} \alpha_{2 l} f_{2 k}^{l}
\end{aligned}
$$

where the $\left\{h_{j}\right\}$ are the impulse response coefficients of the linear FIR filter representing the linear system with memory, the $\left\{\alpha_{1 i}\right\}$ are the parameters of the first nonlinearity, and the $\left\{\alpha_{22}\right\}$ are the parameters of the second nonlinearity.

The $\left\{\hat{h}_{j}\right\},\left\{\hat{\alpha}_{1 i}\right\}$, and $\left\{\hat{\alpha}_{2 l}\right\}$ represent estimates of $\left\{h_{j}\right\},\left\{\alpha_{1 i}\right\}$. and $\left\{\alpha_{2 l}\right\}$ respectively and obey the same relationships as given by Eq. (14). Our objective is to minimize the mean square error $\varepsilon_{k}^{2}$

$$
\varepsilon_{k}^{2}=E\left[\left(e_{k}-\hat{e}_{k}\right)^{2}\right] .
$$

A gradient search algorithm can be used where the instantaneous squared error is taken as an estimate of the true square error. Then the gradient of the instantaneous squared error is found with respect to $\left\{\hat{h}_{j}\right\},\left\{\hat{\alpha}_{1 i}\right\}$, and $\left\{\hat{\alpha}_{2 l}\right\}$. It is easy to verify that the following adaptation equations result

$$
\begin{array}{r}
\hat{\alpha}_{1 i, k+1}=\hat{\alpha}_{1 i, k}+2\left[\sum_{l=0}^{L-1} l \hat{\alpha}_{2 l, k} \hat{f}_{2 k}^{l-1} \sum_{j=0}^{N-1} \hat{h}_{j, k} a_{k-j}^{i}\right] r_{k} \mu_{i}, \\
i=0, \ldots, M-1, \\
\hat{h}_{j, k+1}=\hat{h}_{j, k}+2 \hat{f}_{1, k-j}\left[\sum_{l=0}^{L-1} l \hat{\alpha}_{2 l, k} \hat{f}_{2 k}^{l-1}\right] r_{k} \nu_{j}, \\
j=0, \ldots, N-1, \\
\hat{\alpha}_{2 l, k+1}=\hat{\alpha}_{2 l, k}+2 \hat{f}_{2 k}^{l} r_{k} \xi_{l}, \quad \begin{array}{l}
l \\
\quad=0, \ldots, L-1
\end{array}
\end{array}
$$

where $\mu, \nu$, and $\xi$ represent the stepsizes. The summation $\sum l \hat{\alpha}_{2 l, k} \hat{f}_{2 k}^{l-1}$ is the derivative of the instantaneous estimate of $\hat{e}_{k}$ as given in Eq. (14) (with $e_{k}$ replaced by $\hat{e}_{k}$ ). The advantage of using the nonlinear structure described in this section over a generat nonlinear filter, is that the former requires fewer coefficients

30.3.3. 
than the latter as well as converging faster. For example if the Volterra series nonlınear filter was used to compensate for a nonlınear channel as in Fig. $5,2^{N}$ coefficients would be required. If the new nonlinear structure is used $N+M+L$ coefficients would be necessary, where typically $M+L \leq 8$.

\section{Simulation Results}

In this section computer simulations of the two-memory structure, the combined structure and of the nonlinear compensator are presented. The near-end data and far-end data are uncorrelated \pm 1 values. The echo channel consists of a linear part followed by a static nonlinearity which is then followed by a second linear part. This 3-block model is a general nonlinear model which can be described by the Volterra series representation. The communication channel is also simulated as a 3-block nonlinear model. (The echo and communication channels for the nonlinear compensator are different and will be introduced later). The linear parts are represented by FIR filters. The static nonlinearities are third order polynomials $\left(a_{0}+a_{1}(\cdot)+a_{2}(\cdot)^{2}+a_{3}(\cdot)^{3}\right)$. The ratio of near-end to far-end signal power is $40 \mathrm{~dB}$. The abscissa for all plots is $R_{k}^{2}$ (in $\mathrm{dB}$ ) which is the ratio of residual echo power to far-end data signal power and the ordinate is time in terms of iteration number. The conditions under which each curve is produced ate given within the plots.

\subsection{Simulation Results for the Two-Memory Structure}

The number of bits that address RAM1 (the echo canceller) and RAM2 (the reference-former) is 8 . White Gaussian noise was added to bring the signal-to-noise ratio (SNR) for the communication channel to $20 \mathrm{~dB}$. Fig. 6 compares the theoretical convergence curve using only the echo canceller with the theoretical convergence curve using both the echo canceller and the reference-former (perfect regeneration of the far-end signal is assumed). For curve (a) Eq. (3) is used with $\varepsilon_{n, k}^{2}=\sigma_{u}^{2}$ and a stepsize $\alpha_{C}=0.2$, while for curve (b) Eq. (3) and Eq. (4) are used with $\alpha_{C}=\alpha_{n}=0.2$. In the case where both memories are used, both are activated at the same time. Notice two results. First, the steady state value $R_{\infty}^{2}$ is almost $20 \mathrm{~dB}$ smaller when the reference-former is used. This also implies that if the same steady state value is to be reached, the two-memory structure will converge faster than the case where only one memory is used (i.e. when only the echo canceller is used). This is shown in Fig. 7 where the stepsizes have been chosen to give the same steady state value. Second, the reference-former has little effect while the echo canceller is still converging as can be seen from Fig. 6 where the two curves coincide up to 6,000 iterations. This is due to the fact that while the echo canceller is converging the level of echo is still much higher than the received signal so that the control signal for the reference-former consists mostly of echo. When the echo canceller has reduced the level of the echo to below the received signal, the reference-former begins to converge at a faster rate.

The steady state value $R_{\infty}^{2}$ using Eq. (6) is

$$
R_{\infty}^{2}=\frac{\alpha_{c} \sigma_{n}^{2}}{\left(2-\alpha_{c}-\alpha_{R}\right) \sigma_{u}^{2}},
$$

and under the above conditions $R_{\infty}^{2}=-29.32 \mathrm{~dB}$ for curve (b).

In all of the above cases (where only theoretical curves were considered) both memories were activated from the beginning and perfect regeneration of the far-end signal was assumed. If in a startup situation, adaptation of the two memories (starting initially at zero values) is to be achieved without requiring a prearranged "ideal reference" data sequence, it is desirable to activate the referenceformer after the echo canceller has converged. At the point in time that most of the decisions are correct, the reference-former can be started with a large step size to achieve rapid convergence (the steady state value is not affected very much by the large stepsize).
We now compare computer simulations with the theoretical results. In Fig. 8 the reference-former is activated at iteration 60,000 (the "noisy" curves represent the computer simulations). The simulation results agree well with the theoretical results. Each point in the simulation curves is the sample mean of 100 successive squared errors and normalized by the far-end signal power.

\subsection{Simulation Results for the Combined Structure}

Fig. 9 shows the convergence curve for using a stepsize of $\alpha_{F}=0.005$ for the FIR filter and a stepsize $\alpha_{T}=0.09$ for the RAM. The RAM is activated at iteration 3000 and at that time the coefficients of the FIR filter are 'frozen'.

To see the effect of the FIR filter Fig. 9 also shows the the convergence curve of using only the RAM. The stepsize for the RAM is again $\alpha_{T}=0.09$. The increase in the speed of convergence is considerable. With the FIR filter, steady state is reached at approximately 12,000 iterations whereas without the FIR filter steady state is reached at 22,000 iterations

Fig. 10 shows the convergence curve using Eq. (12) with a stepsize of $\alpha_{F}=0.001$ and the curve obtained from computer simulations. The steady state value of $R_{k}^{2}$ is that predicted by Eq. (13) $\left(R_{\infty}^{2}=0.65 \mathrm{~dB}\right)$. If the stepsize is changed to $\alpha_{F}=0.0007$ there is no apparent improvement in the value of $R_{\infty}^{2}$.

\subsection{Simulation Results for the Nonlinear Compensator}

The nonlinear parts of the echo canceller and of the echo channel are represented by polynomials of order 3 (only second and third order nonlinearities are present). The FIR filter uses 10 coefficients. The communication channel consists only of a linear part, represented by an FIR filter of 6 coefficients. Initially the filter coefficients for the echo canceller and all the nonlinearity parameters are zero except $\hat{\alpha}_{11}$ and $\hat{\alpha}_{21}$ (the linear terms) which are equal to 1 .

The residual echo power to far-end data signal power $R_{k}^{2}$ is plotted against the number of iterations. Eq. (16) is used to adapt the coefficients of the FIR filter and the nonlinearity parameters (with $\mu_{\imath}=\mu, \nu_{j}=\nu, \xi_{l}=\xi$ ). Fig. 11 shows the convergence curve obtained by using $\mu=0.009, \nu=0.05$ and $\xi=0.009$. There are two phases in the convergence curve. The first phase is the time during which the FIR filter converges and is characterized by the sharp decrease of $R_{k}^{2}$ from $40 \mathrm{~dB}$ to about $4 \mathrm{~dB}$. The second phase is the time during which the nonlinear parameters start to have significant values so that they can influence the error and eventually bring it to its minimum value and is characterized by the slow decrease of $R_{k}^{2}$ after about 300 iterations.

\section{Summary and Conclusions}

The problem of nonlinear adaptive filtering for echo cancellation has been considered. Adaptive filter structures which speed up the initial convergence of the conventional table look-up structure have been introduced. One of the structures implements combined echo cancellation and decision feedback equalization. A nonlinear compensator for a specific nonlinear channel model which requires fewer coefficients and converges faster than a general nonlinear filter was also presented. Simulation results were found to agree well with theoretical predictions.

\section{References}

1. N. Holte and S. Stueflotten, "A new digital echo canceller for two-wire subscriber lines", IEEE Transactions on Communications, vol. COM-29, No. 11, pp. 1573-1581, November, 1981.

2. O. Agazzi, D.G. Messerschmitt and D.A. Hodges, “Nonlinear echo cancellation of data signals", IEEE Transactions on Communications, vol. COM-30, No. 11, pp. 2421-2433, November, 1982. 
3. Ernst G. Peter, 'Multistage RAM: An FIR filter for echo cancellation in a digital two-wire subscriber loop", IEEE Transactions on Circuits and Systems, vol. CAS-34, No. 3, pp. 225-232, March, 1987

4. D.D. Falconer, "Adaptive reference echo cancellation". IEEE Transactions on Communications, vol. COM-30, No 9, PP. 2083-2094, September, 1982

5. J. F. Michaelides, "Nonlinear Adaptive Filtering for Echo Can- cellation and Decision Feedback Equalization", $M$. Eng. Thesis, McGill ['mversity, Montreal, Quebec and INRS-Technical Report No. 87-40. Verdun, Quebec, April, 1987.

6. P.V. Gerwen, N A.M. Verhoeckx and T.A.C.M. Claasen, "Design considerations for a $144 \mathrm{kbits} / \mathrm{s}$ digital transmission unit for the local telephone network", IEEE Journal on Selected Areas in Communications, vol. SAC-2, No. 2, pp. 314-323, March, 1984.

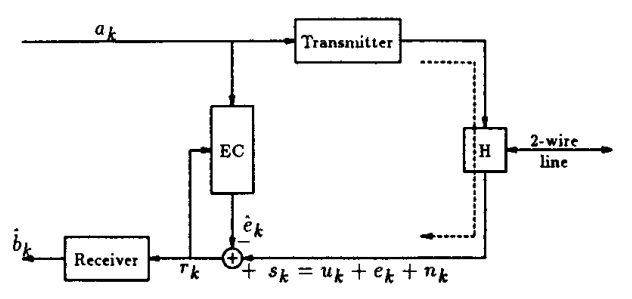

Fig. 1 Echo Cancellation in full-duplex transmission

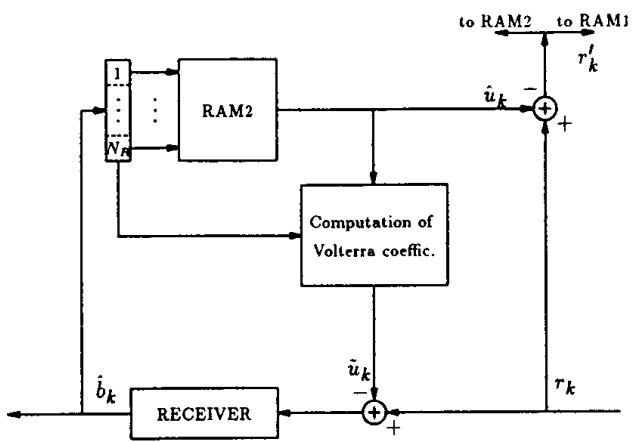

Fig. 3 Decision feedback equalizer structure

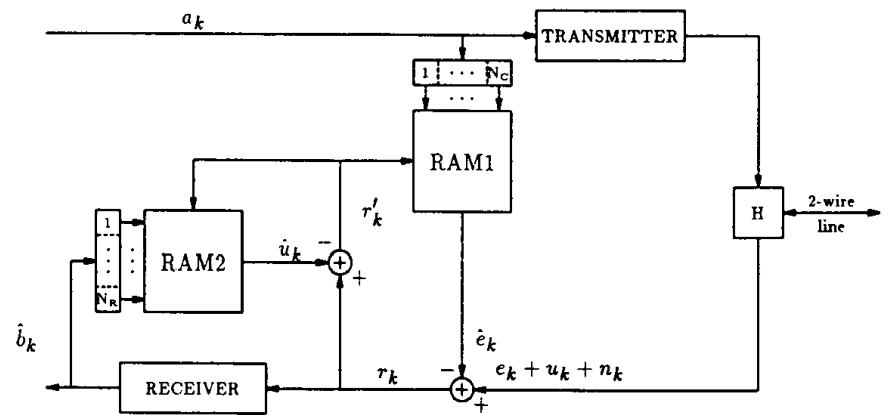

Fig. 2 Adaptive Reference echo cancellation using the table look-up structure

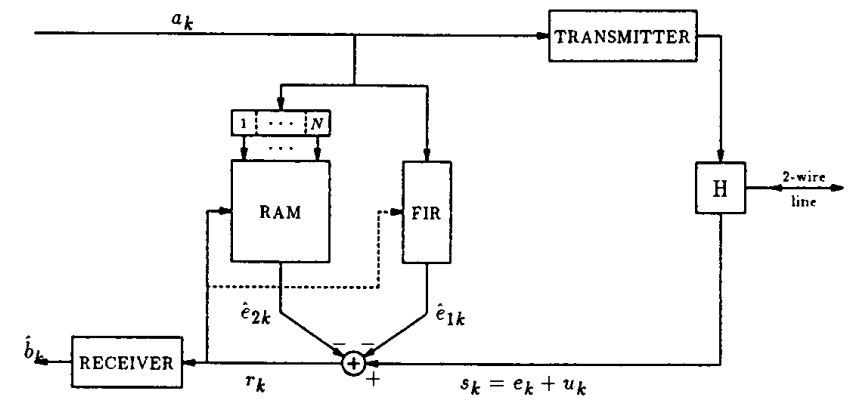

Fig. 4 The Combined Structure

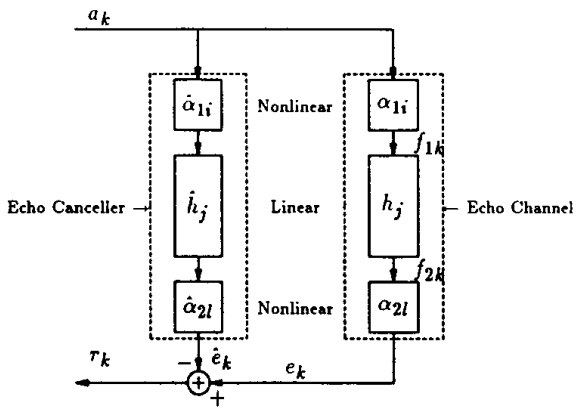

Fig. 5 The new nonlinear compensator

30.3.5. 


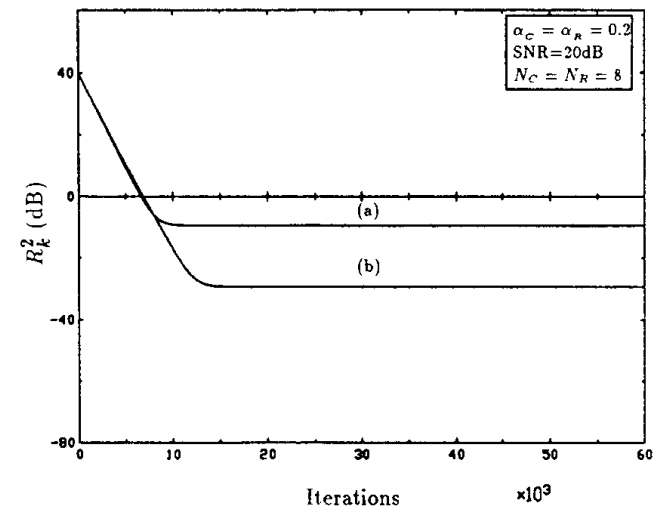

Fig. 6 Theoretical convergence curves using (a) only one memory (the echo canceller alone) and (b) using the two-memory structure

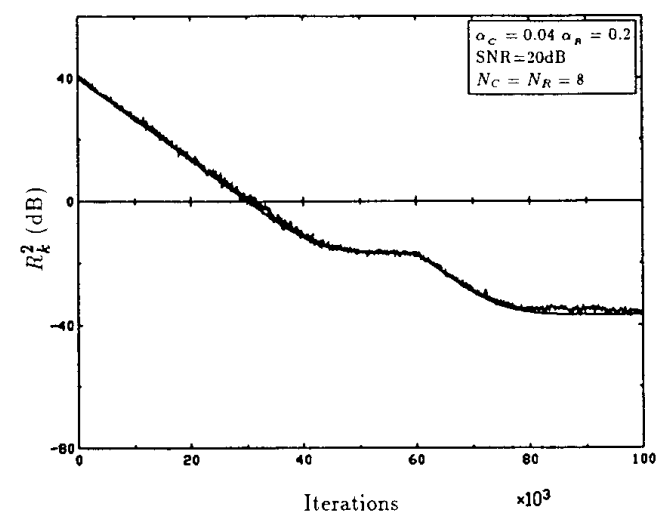

Fig. 8 Theory vs Simulation for adaptive reference echo cancellation (the reference-former is activated at iteration $60,000)$

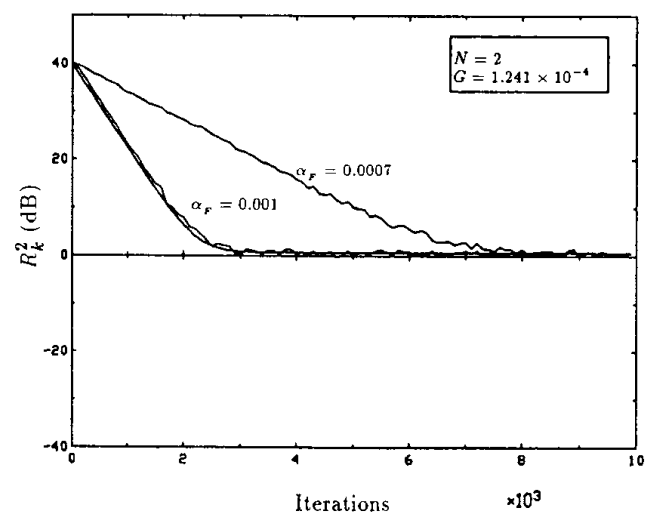

Fig. 10 Theoretical convergence curve vs Computer simulations for a linear FIR filter operating under a nonlinear channel

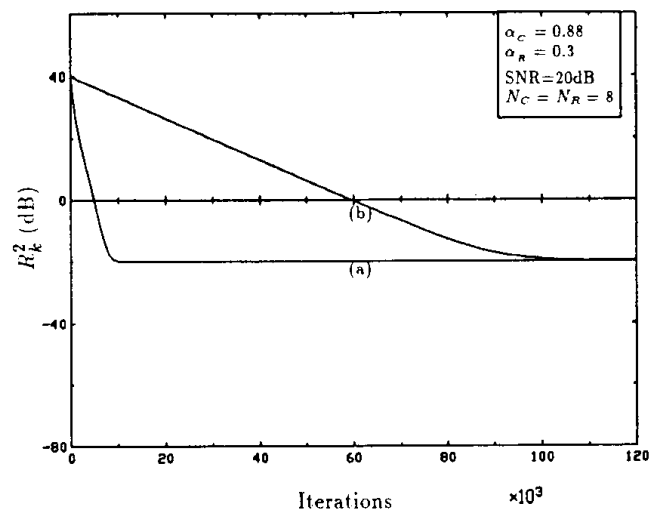

Fig. 7 Comparison of the speed of convergence between (a) the two-memory structure and (b) the case when only one memory is used (the echo canceller, with $\alpha_{C}=0.02$ )

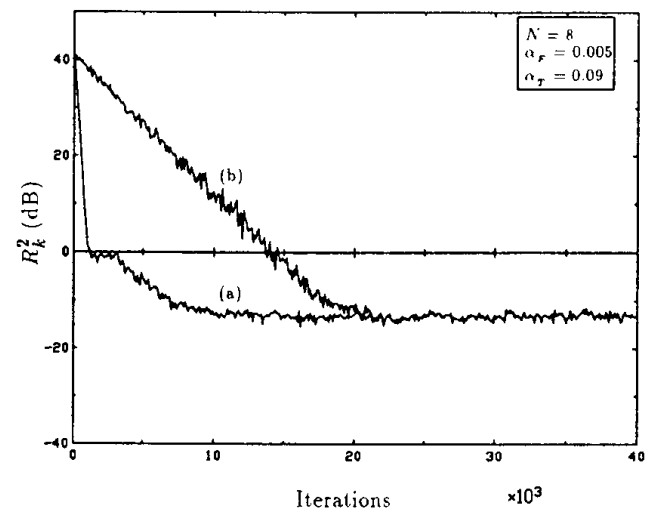

Fig. 9 Combined structure (a) vs Table look-up structure (b)

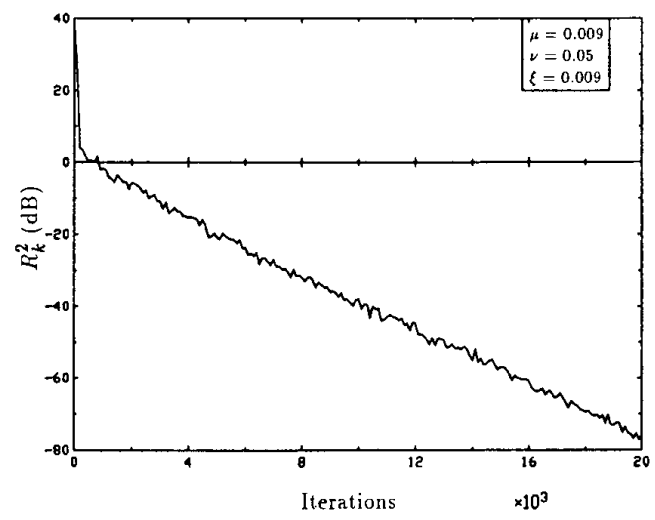

Fig. I1 Convergence curve for nonlinear echo canceller (computer simulation)

30.3.6. 